\title{
Efficient Emission from InAlGaAs Single Quantum Dots with Low Lattice Misfit and AlGaAs Indirect Bandgap Barrier
}

\author{
T. SŁupiński*, K.P. Korona, J. PApierska And J. Borysiuk \\ Faculty of Physics, University of Warsaw, L. Pasteura 5, 02-093 Warsaw, Poland
}

\begin{abstract}
We report on molecular beam epitaxy growth and properties of rarely studied quaternary $\mathrm{In}_{0.4}\left(\mathrm{Al}_{0.75} \mathrm{Ga}_{0.25}\right)_{0.6} \mathrm{As}$ self-assembled quantum dots, which show strong and efficient emission of red light from single quantum dots. The increased yield is, among others, due to efficient energy transfer from indirect band-gap $\mathrm{Al}_{0.75} \mathrm{Ga}_{0.25} \mathrm{As}$ barriers. To maximize photon energy emitted from quantum dots, low In composition, $x_{\text {In }}=0.4$ was applied, which also lowered the lattice misfit close to the limit of 2D/3D transition in the Stranski-Krastanov growth mode. Time-resolved micro-photoluminescence shows emission at $650-750 \mathrm{~nm}$. Well-resolved single quantum dot photoluminescence lines (decay time of $\approx 1-2 \mathrm{~ns}$ ) are observed despite a high concentration $\approx 3 \times 10^{11} \mathrm{~cm}^{-2}$ of quantum dots. We discuss this observation assuming newly a role of carriers or excitons diffusion/tunneling between quantum dots at high surface concentration of dots and a possible role of lattice disorder inside the dot on the exciton lifetime.
\end{abstract}

DOI: 10.12693/APhysPolA.130.1229

PACS/topics: 78.55.Cr, 73.22.-f, 78.47.jd

\section{Introduction}

Quantum dots (QD) formed spontaneously during epitaxy of semiconductor strained layers, or self-organized, coherent with the surrounding crystal lattice, are studied for over 20 years due to a very rich physics of elementary excitations or materials organization, e.g. [1-3]. They are applied in semiconductor lasers or light detectors, and are considered as model single photon sources. The mostly studied in III-V material system are InAs or InGaAs QDs within GaAs or AlGaAs energy barriers. Application of $\mathrm{Al}$ in both QDs and barriers, reported here, allows for a significant modification of energy band structure comparing to InGaAs or GaAs, and shifts exciton emission to higher energy for e.g. single photon sources in the visible range $[4,5]$ or tuning emitters in microcavities, e.g. [6].

In this report, we present molecular beam epitaxy (MBE) growth and properties of quaternary $\mathrm{In}_{0.4}\left(\mathrm{Al}_{0.75} \mathrm{Ga}_{0.25}\right)_{0.6} \mathrm{As}$ QDs embedded in indirect bandgap $\mathrm{Al}_{0.75} \mathrm{Ga}_{0.25} \mathrm{As}$ barriers, a system with much lower lattice misfit comparing to InAs/GaAs. Formation of QDs during epitaxy is a complex process driven by an energy gain when a thick strained layer undergoes the $2 \mathrm{D} / 3 \mathrm{D}$ transition on the free surface. It is strongly influenced by the kinetics [3]. We discuss MBE parameters allowing for efficient emission of red light from single QDs in a dense array of QDs, assuming excitons diffusion/tunneling to preferred QDs for some optimal arrangement of QDs and a possible role of lattice disorder.

\section{Experimental}

We have prepared by solid-source MBE a series of samples containing quaternary $\operatorname{In}_{1-x}\left(\mathrm{Al}_{y} \mathrm{Ga}_{1-y}\right)_{x} \mathrm{As}$

\footnotetext{
* corresponding author; e-mail: Tomasz.Slupinski@fuw.edu.pl
}

self-assembled QDs embedded in $\mathrm{Al}_{y} \mathrm{Ga}_{1-y} \mathrm{As}$ barrier with low In $(1-x=0.4)$ and high Al $(y=0.75)$ content grown on GaAs (001) substrates. For the purpose to maximize photon emission energy from single QDs, the In content was chosen close to the low limit of self-assembled QDs formation in the Stranski-Krastanov (S-K) growth mode, usually reported as $1-x \approx 0.3[7]$. First, GaAs $\approx 300 \mathrm{~nm}$ thick buffer layer was grown at $580^{\circ} \mathrm{C}$, then $100 \mathrm{~nm} \mathrm{Al}_{0.75} \mathrm{Ga}_{0.25} \mathrm{As}$ cladding layer of indirect energy barrier was deposited, half thickness at $580^{\circ} \mathrm{C}$ and the other half at $500^{\circ} \mathrm{C}$. Next, quaternary $\mathrm{In}_{1-x}\left(\mathrm{Al}_{y} \mathrm{Ga}_{1-y}\right)_{x}$ As layer was deposited at $500^{\circ} \mathrm{C}$, which formed $3 \mathrm{D}$ islands, or QDs, and was then capped by top cladding layer of $50 \mathrm{~nm} \mathrm{Al}_{0.75} \mathrm{Ga}_{0.25} \mathrm{As}$ energy barrier followed by $5 \mathrm{~nm}$ layer of GaAs. Finally, for the purpose of atomic force microscopy (AFM) studies, the second layer of $\operatorname{In}_{1-x}\left(\mathrm{Al}_{y} \mathrm{Ga}_{1-y}\right)_{x}$ As forming uncapped 3D islands was deposited in nominally the same conditions as the first layer of QDs. The substrate was then cooled down fast by switching the heater's power off. For all the samples prepared, the conditions determining the diffusion of atoms deposited on the surface for QDs formation, namely temperature of GaAs substrate and As flux intensity, were kept constant, to assure similar conditions of diffusion during QDs nucleation and growth in both InAlGaAs layers and in different samples. The deposition rate of InAlGaAs which form QDs was chosen at high values $0.2-0.8 \mathrm{ML} / \mathrm{s}$. In a series of samples containing InAlGaAs QDs, mainly one parameter in MBE growth was varied - the thickness of deposited InAlGaAs layer, in the range 6-10 ML. At thickness of about $7 \mathrm{ML}$ for $\mathrm{In}_{0.4}\left(\mathrm{Al}_{0.75} \mathrm{Ga}_{0.25}\right)_{0.6}$ As material composition, 2D/3D growth mode change (onset of QDs) was observed by electron diffraction (RHEED) during MBE growth. The embedded layer of InAlGaAs QD was grown without growth interruptions, i.e. was immediately covered with AlGaAs cladding layer, to keep the lens-type shape 
of QDs, since it is established that prolonged annealing of QDs changes the shape of 3D islands to higher prismatictype QDs and, consequently, lowers the energy of quantum confinement and increases the wavelength of emitted photons.

For a comparison purposes, samples with InAs QDs in GaAs barrier, having similar sequence of layers, were also prepared at lower QD growth temperature $470{ }^{\circ} \mathrm{C}$. For critical thickness 1.7 ML of InAs, 2D/3D S-K transition was observed by RHEED.

Micro-photoluminescence was measured at temperatures $T=4-10 \mathrm{~K}$. Time-resolved PL (TRPL) was measured with streak camera. The collecting spot had diameter of about $1 \mu \mathrm{m}$ and $3 \mu \mathrm{m}$ for continuous and TRPL spectroscopy, respectively.

Structural characterization includes atomic force microscopy (AFM) of uncapped islands and cross-section transmission electron microscopy (TEM).

\section{Results and discussion}

According to QDs growth models, e.g. [7-9], QDs formation occurs via nucleation and growth mode. Initial concentration of QDs formed at the $2 \mathrm{D} / 3 \mathrm{D}$ transition critical thickness, $h_{\text {crit }}$, for a relatively small excess thickness of deposited material, $h-h_{\text {crit }}$, is determined mostly by kinetic factors for atoms adsorbed on the surface (adatoms). In Fig. 1, we present AFM picture of uncapped InAs/GaAs samples showing the initial role of surface kinetics. For a small value of the deposition rate (In flux intensity) $0.0037 \mathrm{ML} / \mathrm{s}$ (Fig. 1a), small concentration $\approx 5 \times 10^{9} \mathrm{~cm}^{-2}$ of large $\approx 50 \mathrm{~nm}$ lateral size QDs is seen. For $\approx 50$ times higher growth rate of $0.21 \mathrm{ML} / \mathrm{s}$
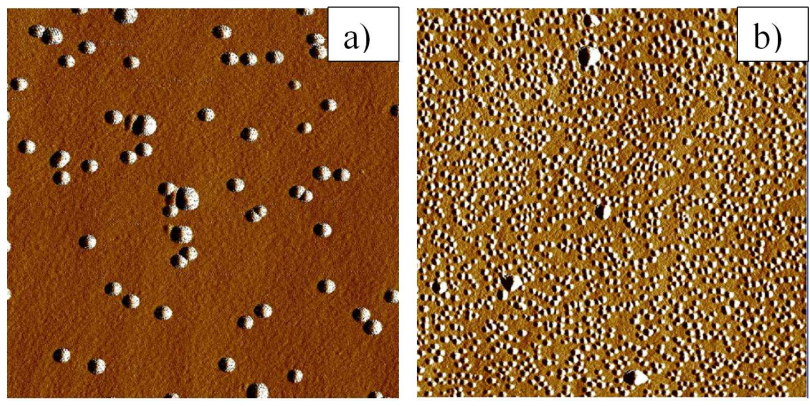

Fig. 1. AFM images $(1 \mu \mathrm{m} \times 1 \mu \mathrm{m})$ of uncapped InAs QDs on GaAs surface showing the role of surface kinetics for initial nucleation and growth of QDs. (a) $1.78 \mathrm{ML}$ of InAs deposited at very low deposition rate $0.0037 \mathrm{ML} / \mathrm{s}$, (b) $1.85 \mathrm{ML}$ of InAs deposited at $0.21 \mathrm{ML} / \mathrm{s}$. Low growth rate allows for long diffusion length of adatoms and results in a small concentration of large QDs.

(Fig. 1b) a larger concentration $\approx 1 \times 10^{11} \mathrm{~cm}^{-2}$ of smaller QDs is seen. This comparison clearly confirms a role of surface kinetics of adatoms diffusion for the initial nucleation of QDs. The two processes compete: (1) new dots nucleation after locally overcoming of $h_{\text {crit }}$ by the deposition thickness and (2) size increase of nucleated dots by the capture of adatoms diffusing on the surface, while the diffusion is regarded as stress-driven toward the existing dots [8]. The dependence of QDs size on the growth rate $V$ observed in Fig. 1 seems however to be weaker than $\sim V^{-1 / 2}$ calculated in model [8], and rather is closer to $\sim V^{-1 / 3}$ mentioned in [9] for the Ostwald ripening.

The micro-photoluminescence of the sample with $\mathrm{In}_{0.4}\left(\mathrm{Al}_{0.75} \mathrm{Ga}_{0.25}\right)_{0.6} \mathrm{As}$ QDs and indirect bandgap $\mathrm{Al}_{0.75} \mathrm{Ga}_{0.25} \mathrm{As}$ barrier (see Fig. 2) reveals strong well resolved lines from single QDs. The estimated surface density of light-emitting QDs is about $50 / \mu \mathrm{m}^{2}$. The AFM of uncapped InAlGaAs layer (see inset of Fig. 2) reveals however a much higher concentration of $3 \mathrm{D}$ islands $\approx 3 \times 10^{11} \mathrm{~cm}=3000 \mu \mathrm{m}^{-2}$. Average lateral size of dots is $20-25 \mathrm{~nm}$ and the height $\approx 2-3 \mathrm{~nm}$, as estimated by RHEED from the time of QDs overgrowth by subsequent layer of AlGaAs barrier.

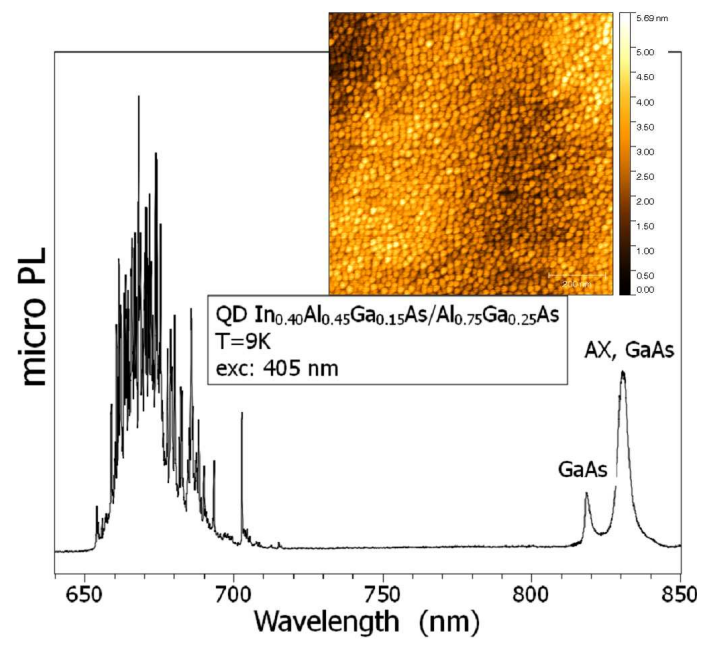

Fig. 2. Micro-PL of QDs $\mathrm{In}_{0.40} \mathrm{Al}_{0.45} \mathrm{Ga}_{0.15} \mathrm{As} / \mathrm{Al}_{0.75} \mathrm{Ga}_{0.25} \mathrm{As}$ formed in $9.0 \mathrm{ML}$ of deposited material. Strong single QDs lines are observed in wavelength range of $650-700 \mathrm{~nm}$. Inset $(1 \mu \mathrm{m} \times 1 \mu \mathrm{m})$ shows AFM picture of uncapped QDs layer in the same sample.

During formation of QDs, the total concentration of QDs increases quickly with the thickness of deposited material, and then the size of dots seems to be determined by energetics rather than by kinetics for the concentration range of $\approx 10^{11} \mathrm{~cm}^{-2}$, as e.g. seen in inset in Fig. 2 . We have found that efficient emission from single QDs could be observed when high surface concentration of dots was formed at layer thickness deposited $\approx 9.0 \mathrm{ML}$, that is when excess $\approx 2 \mathrm{ML} \operatorname{In}_{0.4}\left(\mathrm{Al}_{0.75} \mathrm{Ga}_{0.25}\right)_{0.6} \mathrm{As}$ over the critical thickness $h_{\text {crit }}=7.0 \mathrm{ML}$ is deposited and when QDs layer was grown with high growth rate of $0.2-0.8 \mathrm{ML}$. The wetting layer is thick in this case due to a small lattice misfit $\approx 3 \%$, comparing to $\approx 7 \%$ in InAs/GaAs QDs. We suppose that a thick wetting layer may help in efficient energy transfer from absorbing $\mathrm{Al}_{0.75} \mathrm{Ga}_{0.25} \mathrm{As}$ energy barrier. Another factor which influences a strong single QD emission seems to be an indirect bandgap in AlGaAs barrier, which reduces photon emission in the barrier excited by laser light. 
Comparing to InAs/GaAs QDs system widely studied in the literature, we have noticed that a lowered lattice misfit had resulted in a relatively wider range of epitaxial growth conditions of coherent (pseudomorphic) QDs. The deposited thickness with no dislocations generation in $\operatorname{In}_{0.4}\left(\mathrm{Al}_{0.75} \mathrm{Ga}_{0.25}\right)_{0.6} \mathrm{As} / \mathrm{Al}_{0.75} \mathrm{Ga}_{0.25} \mathrm{As}$ QDs extends to about 3-4 ML above the critical thickness and allows to easily tune the concentration of QDs in a much wider range than in InAs/GaAs, where such a thickness only about $0.5 \mathrm{ML}$ above $h_{\text {crit }}$ is possible, according to our experience.

The emission wavelength of single QD, 1.7$1.85 \mathrm{eV}$, is comparable to $\Gamma$-point energy bandgap of $\operatorname{In}_{0.4}\left(\mathrm{Al}_{0.75} \mathrm{Ga}_{0.25}\right)_{0.6} \mathrm{As}$ alloy, $1.7-1.8 \mathrm{eV}$ [10]. However in QDs, the quantum confinement as well as the compressive hydrostatic stress estimated at $\approx 10 \mathrm{kbar}$ in our compressively strained QDs, should both increase the exciton energy. Therefore, the wavelength of emission measured strongly suggests that the QDs are enriched with In over the intended composition based on intensities of metals molecular beams. This should be expected in InAlGaAs alloy, because In adatoms diffusion on the substrate is faster than Ga or Al. Also, consequently, the inhomogeneous composition in the volume of quaternary individual QD may exist due to In segregation on the surface during QDs nucleation and growth.

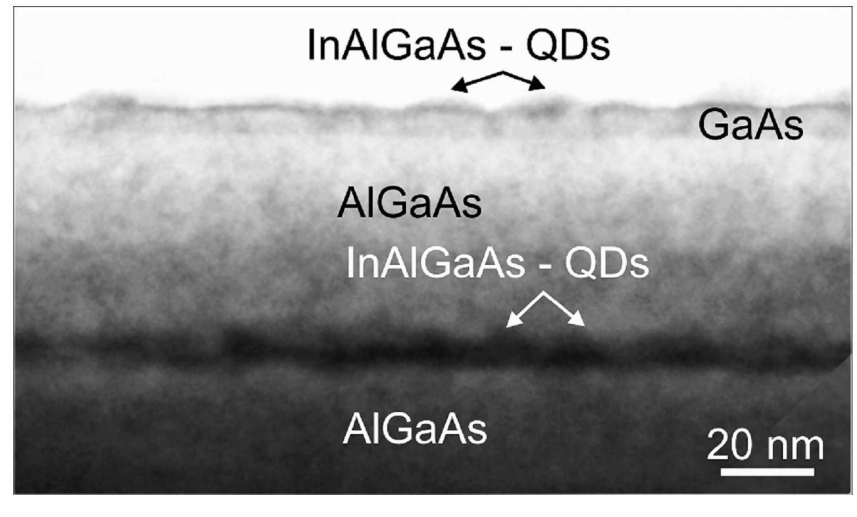

Fig. 3. TEM cross-section image of $\mathrm{In}_{0.4} \mathrm{Al}_{0.45} \mathrm{Ga}_{0.15} \mathrm{As} / \mathrm{Al}_{0.75} \mathrm{Ga}_{0.25} \mathrm{As}$ sample. Similar concentrations of QDs in embedded and uncapped layers can be seen.

To estimate whether the concentration of QDs in embedded layer is comparable to the concentration seen by AFM in uncapped layer, we have made transmission electron microscopy (TEM) studies of sample cross-section - Fig. 3. It may be seen that the number of 3D islands revealed in both embedded and uncapped layers looks similar. This fact is consistent with models of QDs formation in epitaxy mentioned above, where the concentration of dots depends on nucleation conditions and on the total thickness deposited, and these were kept nominally the same in both InAlGaAs layers of 3D islands.

It is important to explain the discrepancy between relatively small number of single QD emission lines seen in
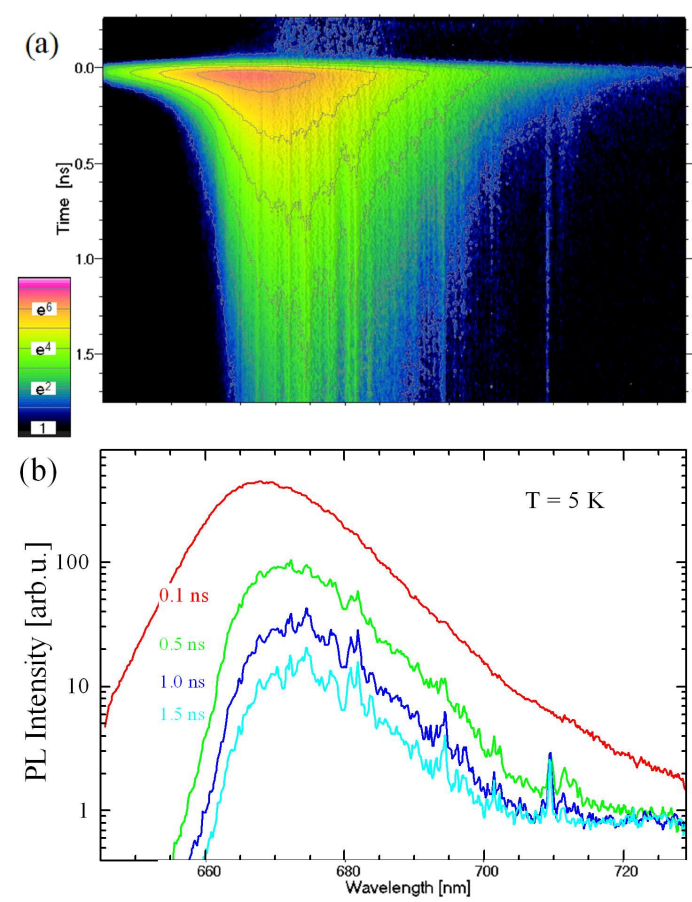

Fig. 4. Time-resolved micro-photoluminescence measurements for sample with $\mathrm{In}_{0.40} \mathrm{Al}_{0.45} \mathrm{Ga}_{0.15} \mathrm{As}$ single QDs. (a) Map of intensity. (b) Intensity at several delay times. Some of single QDs become sharp in time, which indicates that their radiation lifetime is longer than the lifetime of QDs in the broad background.

PL compared to several dozen times higher concentration of 3D islands of QDs seen by AFM in Fig. 2 or TEM in Fig. 3. Some answers can be obtained from time-resolved micro-photoluminescence presented in Fig. 4. The results show single dot emission lines with decay time of 1-2 ns and a broad continuum of lines showing a shorter decay time. In some samples, the radiative lifetime of some single QDs was even as long as $3 \mathrm{~ns}$. The broad continuum probably comes from unresolved individual QDs having shorter radiative lifetime. These observations may indicate several possibilities. The most obvious is that shortliving continuum comes from dots with some recombination centers, e.g. some lattice disorder in QD. Another explanation takes into account that excitons can diffuse or tunnel between different QDs so they can find the QD with locally the lowest energy [11]. Since there is no exciton escape possibility from such dots, they have the longest lifetimes. One would expect that a certain arrangement of dots on the surface (e.g. shorter distance between separated dots) would optimize the probability of excitons tunneling. Interestingly, this last hypothesis could justify a relatively narrow range of MBE growth parameters for observation of well-separated and strong single QD emission lines as a function of MBE growth parameters. In our case of InAlGaAs/AlGaAs, single QD emission lines were observed in samples grown with a relatively narrow range of material thickness deposited, $h / h_{\text {crit }}=1.25-1.30$ (having other important growth parameters fixed). 
Additionally, excitons traveling between neighboring dots could have momentum, and if they couple to the lattice they might emit phonons. This could lead to a broadening of "zero phonon" emission lines in a similar manner like it is discussed to interpret the broadening of single dot emission line increasing temperature, which includes the coupling to acoustic phonons [12, 13]. Interesting possibility seems to be a role of disorder QD's crystal lattice for the strength of exciton-phonon coupling. Such a role could differentiate individual QDs.

Also, the question which may be interesting is what type of disorder in QDs could be the origin of observed shorter radiative lifetime in a large fraction of quaternary QDs present in the QD layer (static lattice disorder e.g. local distortions of QD crystal lattice, or electrostatic one due to e.g. fluctuations of residual impurities). Yet another possibility to describe mentioned QDs concentration discrepancy is that long-lifetime dots may be formed inside a few monolayer thick wetting layer in case of low lattice misfit InAlGaAs/AlGaAs, similar to natural InGaAs QDs reported e.g. in [14].

\section{Conclusions}

We have prepared MBE samples containing $\mathrm{In}_{0.4}\left(\mathrm{Al}_{0.75} \mathrm{Ga}_{0.25}\right)_{0.6} \mathrm{As}$ QDs in indirect bandgap $\mathrm{Al}_{0.75} \mathrm{Ga}_{0.25} \mathrm{As}$ barrier, which show strong and efficient single quantum dot emission in the red range 650$750 \mathrm{~nm}$. We have described MBE growth parameters, which seems to be important to achieve efficient emission from single QDs. Low lattice misfit allows extending the range of $\mathrm{MBE}$ growth parameters for lattice-coherent QDs, but single QD emission lines are observed for still rather narrow range of growth parameters. We have noticed from AFM studies and based on established models of QDs nucleation and growth that the surface density of QDs seems to be significantly higher than the number of single dot emission lines observed in PL. Single QD emission was observed in a case of high surface concentration of $3 \mathrm{D}$ islands, $\approx 3 \times 10^{11} \mathrm{~cm}^{-2}$. This indicates that only a fraction of $3 \mathrm{D}$ islands gives the strong single QD emission. This observation is discussed assuming a possibility of excitons diffusion/tunneling between neighbor QDs, although an ultimate reason remains unclear.

\section{Acknowledgments}

We thank Dr. J. Suffczyński for help in characterization of the samples by microphotoluminescence. Research was supported by National Research Council (NCN), Poland, grant No. 2012/05/B/ST7/02155.

\section{References}

[1] Single Quantum Dots, Fundamentals, Applications and New Concepts, Ed. P. Michler, Topics in Applied Physics, Vol. 90, Springer, Berlin 2003.

[2] Quantum Dots: Fundamentals, Applications, and Frontiers, Eds. B.A. Joyce, P.C. Kelires, A.G. Naumovets, D.D. Vvedensky, NATO Science Series, Mathematics, Physics and Chemistry, Vol. 190, Springer, The Netherlands 2005.

[3] V. Shchukin, E. Schöll, P. Kratzer, in: Semiconductor Nanostructures, Ed. D. Bimberg, Springer, Berlin 2008.

[4] T.W. Schlereth, C. Schneider, S. Höfling, A. Forchel, Nanotechnology 19, 045601 (2008).

[5] Ł. Dusanowski, A. Golnik, M. Syperek, M. Nawrocki, G. Sek, J. Misiewicz, T.W. Schlereth, C. Schneider, S. Höfling, M. Kamp, A. Forchel, Appl. Phys. Lett. 101, 103108 (2012).

[6] D. Valente, J. Suffczyński, T. Jakubczyk, A. Dousse, A. Lemaître, I. Sagnes, L. Lanco, P. Voisin, A. Auffeves, P. Senellart, Phys. Rev. B 89, 041302(R) (2014).

[7] Ch. Hayn, Phys. Rev. B 64, 165306 (2001).

[8] V.G. Dubrovskii, G.E. Cirlin, V.M. Ustinov, Phys. Rev. B 68, 075409 (2003).

[9] P. Politi, G. Grenet, A. Marty, A. Ponchet, J. Villain, Phys. Rep. 324, 271 (2000).

[10] I. Vurgaftman, J.R. Meyer, L.R. Ram-Mohan, J. Appl. Phys. 89, 5815 (2001).

[11] K.P. Korona, P. Wojnar, J.A. Gaj, G. Karczewski, J. Kossut, J. Kuhl, Solid State Commun. 133, 369 (2005).

[12] L. Besombes, K. Kheng, L. Marsal, H. Mariette, Phys. Rev. B 63, 155307 (2001).

[13] S. Rudin, T.L. Reinecke, M. Bayer, Phys. Rev. B 74, 161305 (2006).

[14] A. Babiński, J. Borysiuk, S. Kret, M. Czyż, A. Golnik, S. Raymond, Z.R. Wasilewski, Appl. Phys. Lett. 92, 171104 (2008). 